\title{
Tunable nano devices fabricated by controlled deposition of gold nanoparticles via focused ion beam
}

\author{
Asaf Shahmoon $^{\mathrm{a}}$, Ofer Limon ${ }^{\mathrm{a}}$, Olga Girshevitz ${ }^{\mathrm{b}}$, Yafit Fleger $^{\mathrm{b}}$, Hilmi Volkan Demir ${ }^{\mathrm{c}}$, Zeev Zalevsky ${ }^{\mathrm{a}, *}$ \\ ${ }^{a}$ School of Engineering, Institute of Nanotechnology, Bar-Ilan University, Ramat-Gan 52900, Israel \\ ${ }^{\mathrm{b}}$ Nanotechnology Center, Bar-Ilan University, Ramat-Gan 52900, Israel \\ ${ }^{\mathrm{c}}$ Electronics Engineering, Bilkent University, Bilkent, Ankara 06800, Turkey
}

\section{A R T I C L E I N F O}

\section{Article history:}

Received 10 September 2009

Received in revised form 7 December 2009

Accepted 9 December 2009

Available online 16 December 2009

\section{Keywords:}

Particle deposition

Nanoparticle patterning

Nanofabrication

Particle attachment

\begin{abstract}
A B S T R A C T
In this paper, we present the fabrication procedures as well as the preliminary experimental results of a novel method for significantly simplified deposition of charged nanoparticles at specific patterns based on focused ion beam (FIB) technology. The deposition method relies on the implantation of positive gallium ions on an insulated material which creates the basis for attracting the nanoparticles to the substrate. In order to substantiate the theory two patterns were generated on a silicon on insulator (SOI) chip with an upper layer of silicon of $200 \mathrm{~nm}$. The two patterns are as follows: resolution target - consisting of six squares and $400 \mathrm{~nm} \times 400 \mathrm{~nm}$ circular sinusoidal tunnel. In addition, we demonstrate the utilization and applicability of the aforementioned method in a tunable radiation nano device as well as show its experimental characterization.
\end{abstract}

(c) 2009 Elsevier B.V. All rights reserved.

\section{Introduction}

Nanophotonic devices have gained a major interest in the world of engineering and biomedicine during the past few years. The main advantages of this field are in having devices with small size, lower power consumption, higher modulation rate and low cost. Moreover, nanoparticles which are being integrated into a photonic structure enable creation of sophisticated and novel devices due to their unique properties. It is well known that, at nano scale materials behave differently compared to larger scale. The physical and chemical properties of nanoparticles are strongly dependent upon their size, geometries, internal morphological, dielectric constant and their aspect ratio. Nanoparticles can be used in order to block, deflect and manipulate light. Surface plasmon resonance (SPR) [1-3] arises as a response to external electromagnetic radiation which causes the light to be scattered with high efficiency. The prediction of the physical and chemical properties of these nanoparticles remains an intractable challenge and therefore may initiate opportunities for developing novel devices based on these unique properties.

Furthermore, manipulation of nanoparticles with dimension of starting at a few tens and up to a few hundred of nanometers can be used for photonic sensing and modulation applications [4]. Therefore, a mass production process allowing the deposition of the nanoparticles is a prerequisite requirement for those devices.

\footnotetext{
* Corresponding author.

E-mail address: zalevsz@eng.biu.ac.il (Z. Zalevsky).
}

Several methods have been employed to produce deposition of nanoparticles on a surface using the atomic force microscope (AFM) tip in order to apply pulses of voltage into an insulating films $[5,6]$, printing technique that uses electrostatic force to pattern PMMA (polymethyl methacrylate) [7], electrostatic force generated by $p-n$ junction [8] and lithography process that uses conductive flexible poly-dimethyl-siloxane (PDMS) stamp to create charged substrates [9]. Another common method uses a polyL-lysine (PLL) which is a positive organic layer in order to coat the substrate and make an intermediary between the negative nanoparticles and the substrate. The linking between the surface and the poly-L-lysine as well as between the poly-L-lysine and the nanoparticles is based upon electrostatic bonding.

In this paper, we present a novel method for significantly simplified deposition of charged nanoparticles at specific patterns based upon focused ion beam (FIB) technology. This technique is suitable for mass production fabrication and thus may allow large scale realization of various nano photonic modulators as those mentioned by Ref. [4]. The main advantages of this technology are by having both high speed and precision deposition. In this paper, we also discuss the fabrication procedure as well as the preliminary experimental characterization of a tunable device using a trapped nanoparticle.

\section{Patterning method}

The desired patterns were created using FIB with a gallium ions source. Patterning is performed by accelerating concentrated 


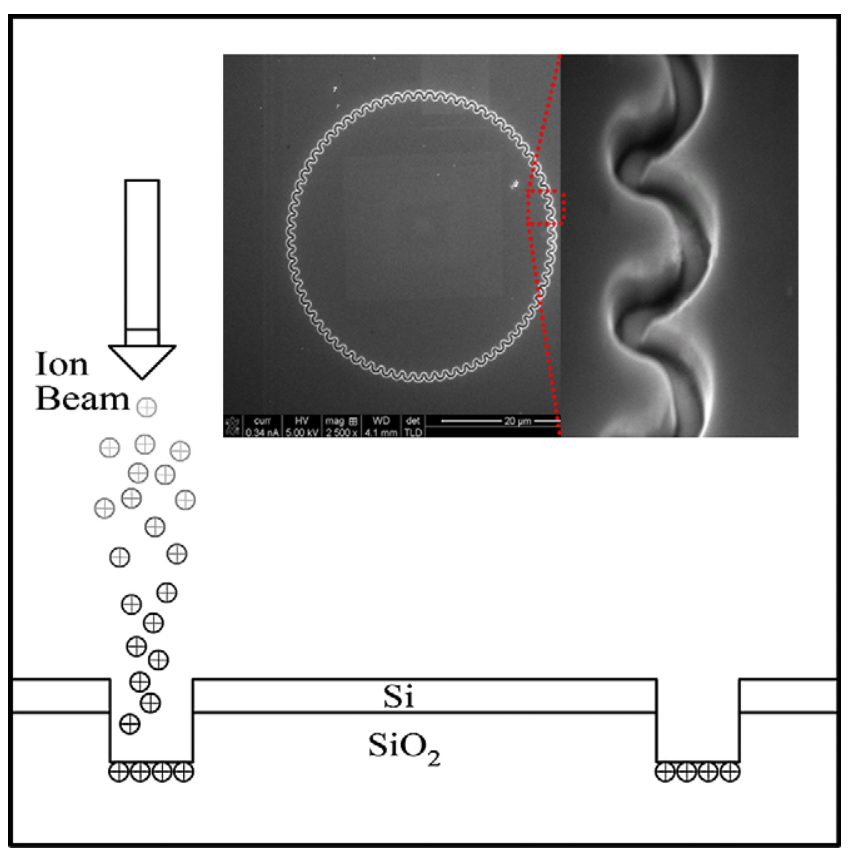

Fig. 1. Schematic sketch of the system - generation of charge pattern using focused ion beam. Inlet shows generation of one of the desired patterns.

gallium ions to a specific area on the chip, which etches off any exposed surface. Positive gallium ions will also be implanted into the top few nanometer of the surface and therefore will create the basis for bonding with the nanoparticles. This trapped positive charge attracts the gold nanoparticles which are negatively charged as part of their fabrication process.

In Fig. 1, we present the schematic sketch of the system in which we created the desired pattern (inlet picture). The imaging resolution and therefore the deposition resolution are determined by the FIB's sputter limited signal to noise ratio of about $6 \mathrm{~nm}$.

The proposed method was experimentally successfully applied to silicon oxide wafers, but can be extended to any other insulated material.

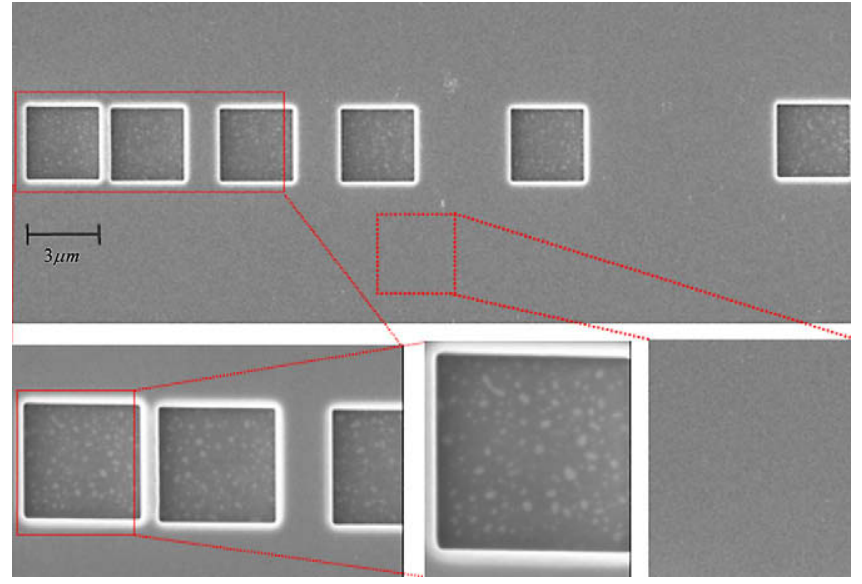

Fig. 2. SEM images of the fabricated resolution target after deposition of $30 \mathrm{~nm}$ gold nanoparticles. The deposition of the nanoparticles was exclusively achieved only at the generated pattern with high precision and contrast.

\section{Experimental result}

In the experiments, we used an unconjugated gold colloidal in an aqueous suspension. The diameters of the particles that we used were $200 \mathrm{~nm}$ and $30 \mathrm{~nm}$ while the standard deviation of the size distribution was less than $10 \%$ of the average.

In Fig. 2, we generated a resolution pattern with different spaces between two adjacent squares. The width of the squares is $3 \mu \mathrm{m}$ with depth of $0.5 \mu \mathrm{m}$. This pattern was created with ion beam having the following parameters: magnification of 2500 , beam current of $0.28 \mathrm{nA}$ and a high voltage of $5 \mathrm{kV}$ following patterning, we deposit $30 \mathrm{~nm}$ gold nanoparticles for $30 \mathrm{~min}$, rinsing with purified HPLC water, drying with nitrogen and finally imaged topographically by scanning electron microscope (SEM). One may see that the deposition of the nano metric particles was exclusively achieved only at the generated pattern with a high level of precision and contrast.

In Fig. 3, we present another successful deposition following a unique $400 \mathrm{~nm} \times 400 \mathrm{~nm}$ circular sinusoidal shape to be later on used as a tunable radiation source. In Fig. 3a and b, we present the AFM topographic images of the device. Fig. 3a presents the a

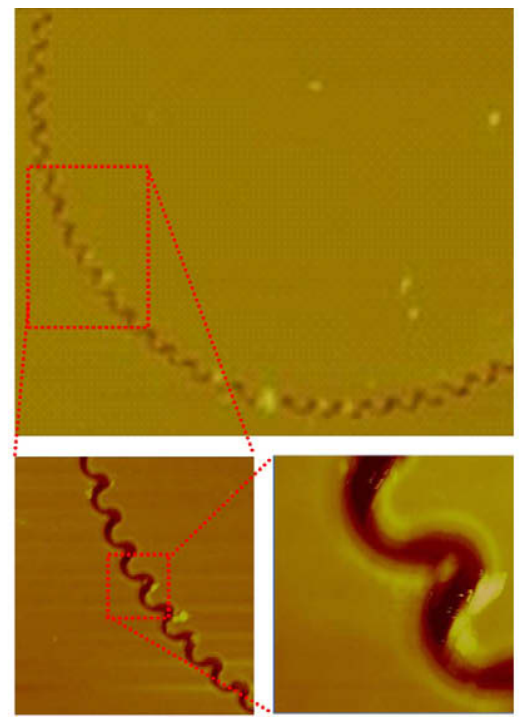

b
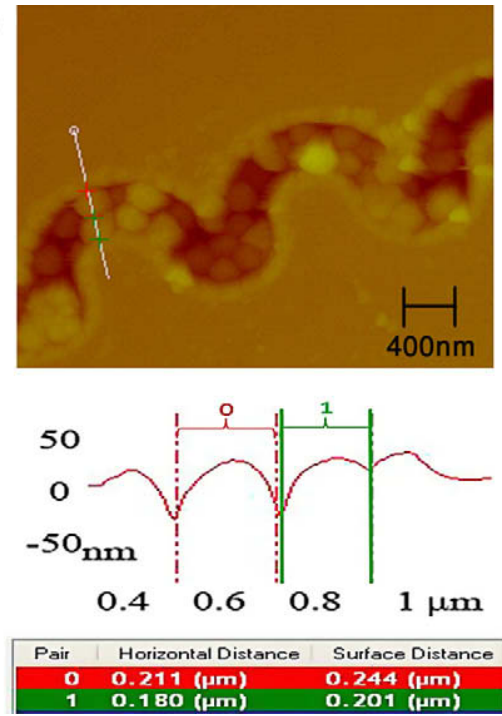

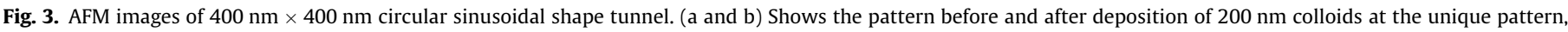
respectively. The lower part of (b) presents a cross section of the tunnel which verifies a pair of $200 \mathrm{~nm}$ particles in the tunnel. 

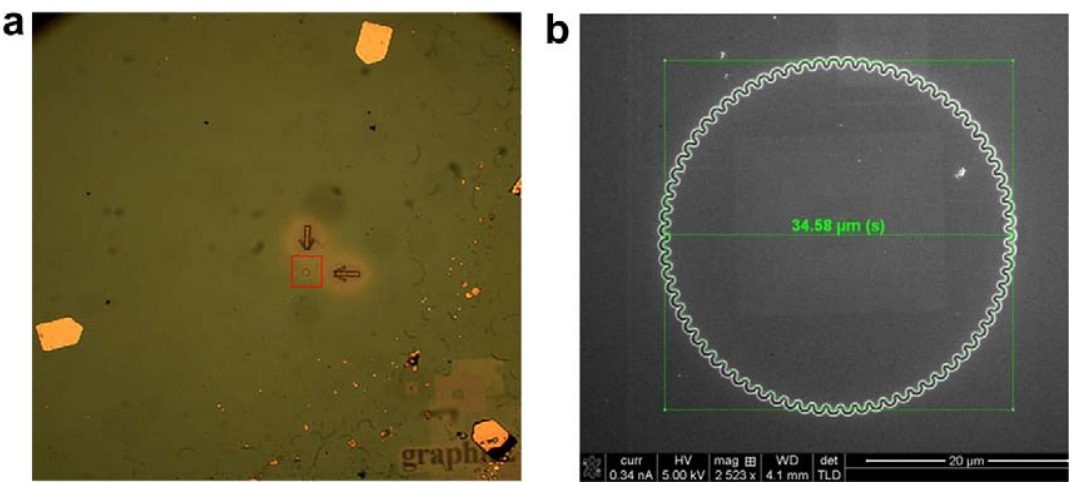

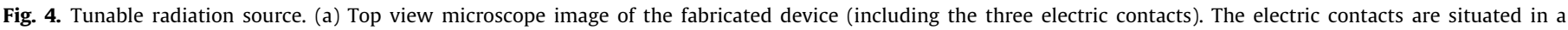
triangular form to enable operation with three phase voltage. (b) SEM image of the encompass area shown in (a) that defines the circular sinusoidal tunnel.

a

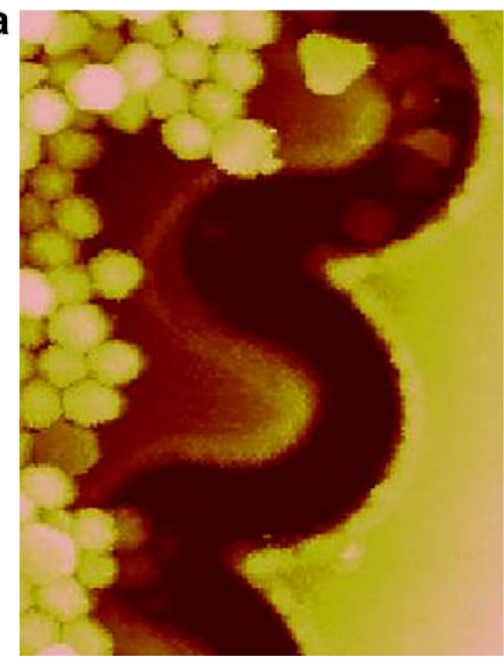

b

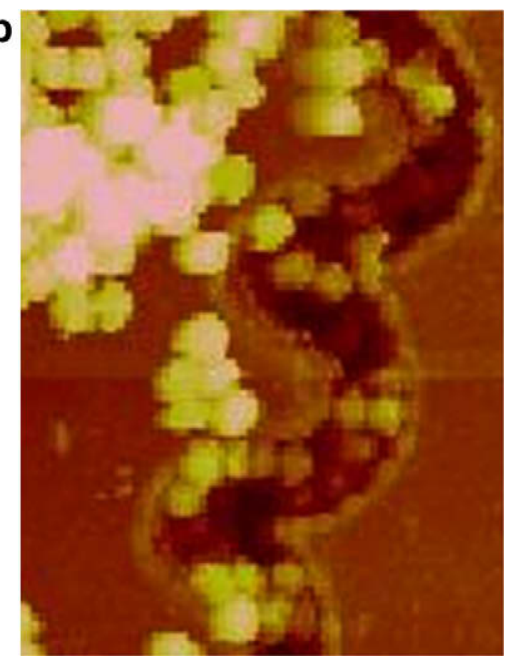

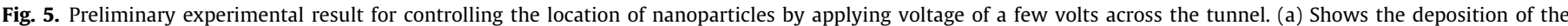

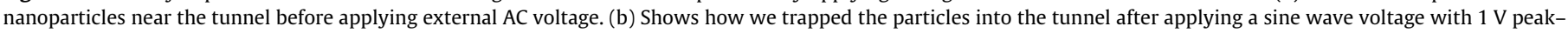
peak and at frequency of $10 \mathrm{kHz}$.

pattern before deposition while the upper part of Fig. 3b demonstrates that indeed $200 \mathrm{~nm}$ colloids were attracted to the pattern. The lower part of Fig. 3b presents a cross section of the tunnel which verifies the existence of a pair of $200 \mathrm{~nm}$ particles in the tunnel. The deposition process was carried out approximately seven months after generating the charge pattern. This passing long period of time is an indication for the high stability and long life time of the implanted gallium ions and thus their applicability for reliable and long term usage in tunable devices such as modulators and sensors.

In Figs. 4 and 5, we present the usage of the proposed mass production fabrication technique for realizing tunable nano devices. Fig. 4 shows the tunable radiation device that was generated using FIB. The operation mechanism of this device is as follows: moving charged nano particle along special tunnel that is causing the particle to periodically accelerate (with positive as well as negative acceleration values), generates radiation. Therefore, this effect can be used for realizing nano scale tunable radiation source. The operation procedure of the device, which is needed for shifting the charged particle along the tunnel, includes connection of an external voltage source to the three electrodes (shown in Fig. 4a) while a delay phase of $2 \pi / 3$ is generated between each two. Fig. 4a present's top view microscope image of the entire fabricated device including the three electric contacts that are linked through wire bonding to the external voltage source. The distance between each contact and the circular sinusoidal tunnel is $1 \mathrm{~mm}$. The electric contacts were generated by a lithography process and by using a negative resist. The contacts were thermally evaporated using $15 \mathrm{~nm} \mathrm{Ti} / 250 \mathrm{~nm}$ Au. Fig. 4b presents SEM image of the encompass area shown in Fig. 4a. The dimensions of the fabricated device were: total diameter of $34.58 \mu \mathrm{m}$, tunnel width of $300 \mathrm{~nm}$ and periodicity of the sinusoidal perturbation of $2.8 \mu \mathrm{m}$. The radiated frequency is proportional to the number of period in the sinusoidal tunnel as seen in Fig. 4b. Wide range of AC voltages can be tested through the external voltage source.

In Fig. 5a and b, we present preliminary experimental result for controlling the location of the nanoparticles (that are positioned inside the circular tunnel) by applying voltage of a few volts across the tunnel. In Fig 5a, we show the deposition of the nanoparticles near the tunnel whereas in Fig. 5b we show how we trapped the particles into the tunnel after applying a sine wave voltage with $1 \mathrm{~V}$ peak-peak and at a frequency of $10 \mathrm{kHz}$.

\section{Conclusion}

We have demonstrated the usage of focused ion beam for patterning of nanoparticles using positive gallium ions as an intermediary between the nanoparticles and the substrate. This unique approach is suitable for mass production process and may allow 
high speed and precise deposition of nanoparticles at nano structure devices that can be used for a wide range of modulation and sensing applications. Usage of the proposed fabrication approach for the realization of a tunable radiation source was demonstrated.

\section{References}

[1] C.J. Murphy, A.M. Gole, S.E. Hunyadi, J.W. Stone, P.N. Sisco, A. Alkilany, B.E. Kinard, P. Hankins, Chem. Commun. 5 (2008) 544-557.

[2] S. Eustis, M.A. El-Sayed, Chem. Inf. 37 (2006) 209-217.
3] P.K. Jain, S. Eustis, M.A. El-Sayed, J. Phys. Chem. B 110 (2006) 18243-18253.

[4] O. Limon, L. Businaro, A. Gerardino, L. Bitton, A. Frydman, Z. Zalevsky, Microelectron. Eng. 86 (2009) 1099-1102.

[5] P. Mesquida, A. Stemmer, Microelectron. Eng. 61-62 (2002) 671-674.

[6] N. Naujoks, A. Stemmer, Microelectron. Eng. 67-68 (2003) 736-741.

[7] H.O. Jacobs, S.A. Campbell, M.G. Steward, Adv. Mater. 14 (2002) 1553-1557.

[8] D.H. Tsai, S.H. Kim, T.D. Corrigan, R.J. Phaneuf, M.R. Zachariah, Nanotechnology 16 (2005) 1856.

[9] M. Kang, H. Kim, B. Han, J. Suh, J. Park, M. Choi, Microelectron. Eng. 71 (2004) 229-236. 